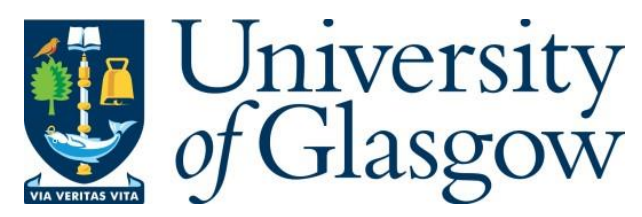

Cortes, E. et al. (2016) Plasmonic nanoprobes for stimulated emission depletion nanoscopy. ACS Nano, 10(11), pp. 10454-10461.

There may be differences between this version and the published version. You are advised to consult the publisher's version if you wish to cite from it.

http://eprints.gla.ac.uk/150500/

Deposited on: 6 November 2017

Enlighten - Research publications by members of the University of Glasgow http://eprints.gla.ac.uk 


\title{
Plasmonic Nanoprobes for Stimulated Emission Depletion Nanoscopy
}

\author{
Emiliano Cortés ${ }^{1 *}$, Paloma A. Huidobro ${ }^{1 *}$, Hugo G. Sinclair ${ }^{2 \ddagger}$, Stina Guldbrand ${ }^{2}$, William J. \\ Peveler ${ }^{3}$, Timothy Davies ${ }^{4}$, Simona Parrinello ${ }^{4}$, Frederik Görlitz², Chris Dunsby, ${ }^{2,5}$, Mark A. A. \\ Neil$^{2}$, Yonatan Sivan ${ }^{6}$, Ivan P. Parkin ${ }^{3}$, Paul M. W. French ${ }^{2}$, Stefan A. Maier ${ }^{1}$
}

1- Experimental Solid State Group, Department of Physics, Imperial College London, SW7 2AZ, UK

2- Photonics Group, Department of Physics, Imperial College London, SW7 2AZ, UK

3- Department of Chemistry, 20 Gordon Street, University College London, WC1H OAJ, UK

4- MRC Clinical Sciences Centre (CSC), Imperial College London, Du Cane Road, London, W12 ONN, UK

5- Centre for Pathology, Imperial College London, Du Cane Road, London, W12 ONN, UK

6- Unit of Electro-optics Engineering, Ben-Gurion University, Beer-Sheba 8410501, Israel

‡ Present address: Institute of Structural and Molecular Biology, Darwin Building, Gower Street, University College London, WC1E 6BT, UK

*Corresponding authors: E.C. e.cortes@imperial.ac.uk and P.A.H p.arroyo-huidobro@imperial.ac.uk

\begin{abstract}
Plasmonic nanoparticles influence the absorption and emission processes of nearby emitters due to local enhancements of the illuminating radiation and the photonic density of states. Here, we use the plasmon resonance of metal nanoparticles in order to enhance the stimulated depletion of excited molecules for super-resolved nanoscopy. We demonstrate stimulated emission depletion (STED) nanoscopy with gold nanorods with a long axis of only $26 \mathrm{~nm}$ and a width of $8 \mathrm{~nm}$ that provide an enhancement of up to $50 \%$ of the resolution compared to fluorescent-only probes without plasmonic components irradiated with the same depletion power. The nanoparticle-assisted STED probes reported here represent $a \sim 2 \times 10^{3}$ reduction in probe volume compared to previously used nanoparticles. Finally, we demonstrate their application toward plasmon-assisted STED cellular imaging at low-depletion powers and we also discuss their current limitations.
\end{abstract}

Key-words: STED nanoscopy, nanorods, super-resolution, plasmonic nanoparticles, bio-imaging 
The diffraction limit has ceased to be a practical limit to resolution in far-field microscopy, following the demonstration of STED, ${ }^{1,2,3}$ RESOLFT $^{4}$ and localisation microscopies ${ }^{5,6,7}$ and the subsequent development of a plethora of super-resolved nanoscopy techniques. ${ }^{8}$ In particular, stimulated emission depletion (STED) nanoscopy, which builds on the advantages of laser scanning confocal microscopy, is a powerful technique for super-resolved imaging in complex biological samples including live organisms. ${ }^{9,10}$ STED nanoscopy uses stimulated emission to turn off the spontaneous fluorescence emission of dye molecules, typically overlapping a focused excitation beam with a "doughnut" shaped beam that deexcites emitters to the ground state everywhere except for the area within the centre of the doughnut, thus providing theoretically diffraction-unlimited resolution in the transverse plane by reducing the fullwidth half-maximum (FWHM) of the point spread function. By increasing the power of the depletion beam the emission region can be can be drastically reduced - theoretically allowing for sub-nanometre resolution - with resolutions of less than $10 \mathrm{~nm}$ being demonstrated. ${ }^{11}$ The scaling of resolution with the square root of the depletion beam power means that relatively high-power lasers are typically used for STED nanoscopy. In practice, however, the use of high-power irradiation can result in problems such as photobleaching of the fluorophores and phototoxicity, and so the achievable resolution is compromised by the need to limit the intensity of the depletion laser radiation. Furthermore, high power lasers can add cost and complexity to STED microscopes and so the requirement for high power depletion beams presents challenges for parallelizing STED measurements ${ }^{12,13}$ in terms of the lasers required, thus, limiting the potential for faster super-resolved imaging.

To some extent the issue of photobleaching can be addressed with the development of more robust fluorescent labels such as quantum dots ${ }^{14}$ as labelling agents but they still require relatively intense depletion-beams and present concerns around phototoxicity. In order to reduce the required intensity of the depletion beam, we recently proposed the use of plasmonic nanoparticles (NPs) whose localized surface plasmon resonances (LSPRs) are spectrally tuned to the depletion beam wavelength. ${ }^{15,16,17}$ Briefly, materials that present plasmonic resonances enable an intense sub-wavelength concentration of light to below the diffraction limit, mediating electromagnetic (EM) energy transfer either from the far to the near field or vice versa. Thus, these elements can be considered as optical nanoantennas and are key instruments in the conversion of free-space light to nanometre-scale volumes. ${ }^{18,19}$ For metal NPs at their LSPR, their scattering cross section can be much larger than their physical cross section, which allows them to efficiently capture light. This light is then concentrated in the vicinity of the nanoparticle and can be used, for example, to deplete excited fluorescent molecules, among others. ${ }^{20,21,22,23}$ Thus, if the LSPR of the metal NP is centred at the depletion-laser wavelength, the input depletion-power requirements for STED microscopy can be met at much lower intensities than normally used.

Our recent demonstration of the proof of concept of nanoparticle-assisted STED (NP-STED) nanoscopy utilized $160 \mathrm{~nm}$ core@shell (silica@Au) spherical particles, with a plasmon resonance at $800 \mathrm{~nm}$ and fluorescent dyes embedded in the silica core. ${ }^{17}$ However, such large NPs are not practically useful for most studies in biology. In order to facilitate their incorporation into cells and to best represent the underlying labeled structures of interest when using them to read out biological processes, the size of the plasmonic-fluorescent probes should be as small as possible. ${ }^{24}$ Typical resolution achieved in STED using fluorescent dyes for bio-imaging is below $50 \mathrm{~nm}$, which can be taken as an upper limit for the sizes 
of experimentally useful plasmonic NP labels. ${ }^{25} \mathrm{~A}$ further issue for the core@shell nanoparticles used in our proof of concept demonstration was the observation of unwanted background light arising from gold luminescence. This limited the resolution achievable such that it was necessary to implement timegated detection in order to supress this unwanted background light. ${ }^{17}$

For NP-STED microscopy implemented with a mode-locked Ti:Sapphire laser to provide the depletion radiation, plasmonic-probes utilising the fluorescence of the most popular near infrared STED dyes should be designed to have a plasmon resonance at $\sim 80 \mathrm{~nm}$ and ideally be smaller than $50 \mathrm{~nm}$. Au and $\mathrm{Ag}$ are the most common materials employed in plasmonics to achieve near-infrared and visiblewavelength LSPRs, due to their well-behaved optical constants. ${ }^{26}$ By tuning the shape and size of the particles it is possible to shift their plasmon resonance over the whole visible and the near-infrared spectrum. ${ }^{27}$ However, as Ag and Au spheres of $\sim 50 \mathrm{~nm}$ diameter have their plasmon resonances at $\sim 450$ and $\sim 550 \mathrm{~nm}$ respectively, achieving a LSPR at $780 \mathrm{~nm}$ with a $50 \mathrm{~nm}$ maximum-size for metallic (Ag or $\mathrm{Au}$ ) particles requires the use of non-spherical particles. Anisotropic particles, such as gold nanorods (AuNRs), emerge as a suitable shape to fulfil these requirements as their plasmon resonance can be tuned by adjusting their aspect ratio (length/width). ${ }^{28}$ Specifically, AuNRs with an aspect ratio of 3.25 ensure a longitudinal plasmon resonance at $780 \mathrm{~nm}$ in oil; usually used as a refractive index matching media for microscopy measurements. Thus, if the length and width of the AuNRs are reduced while keeping this ratio constant, it is possible to achieve smaller plasmonic-probes suitable for STED nanoscopy. ${ }^{29}$

Here, we demonstrate this extension of NP-STED to smaller, anisotropic particles by synthesizing plasmonic-probes based on $26 \times 8 \mathrm{~nm}$ fluorescently-labelled AuNRs and show that we can achieve a resolution improvement of $\sim 50 \%$ using STED microscopy at low depletion intensities $\left(1.5 \mathrm{MW} / \mathrm{cm}^{2}\right)$ for which a control experiment using fluorescent beads without plasmonic enhancement presents a much weaker $(<10 \%)$ improvement in resolution. The plasmonic nanoprobes for STED reported here are $\sim 2000$ times smaller in volume compared to the nanoparticles we reported previously ${ }^{17}$ and we have used them to label adult neural stem cells for STED nanoscopy at low depletion powers. We also note that the reduction $(\sim 1000 \mathrm{x})$ in the amount of metal of these NP means that we no longer detect the unwanted background light from gold luminescence.

\section{Results and Discussion:}

We start by describing the functionalization and characterization of the small plasmonic probes, designed to perform as labelling agents for low-power STED super-resolution imaging. As already mentioned, we synthesized small, fluorophore-coated AuNRs, whose longitudinal plasmon resonance is matched with the depletion-beam wavelength (780 nm in our STED microscope, see Figure S1 for more information about the STED-FLIM set-up). In order to conjugate the fluorescent dye (STAR 635P) onto the surface of the AuNRs we used a biotin-streptavidin system (Figure 1(a)). This linker provides a separation of $\sim 5 \mathrm{~nm}$ between the fluorophore and the metal surface of the nanorods in order to avoid fluorescence quenching. ${ }^{30,31}$ It also enables us to incorporate a molecule providing chemically specific 
binding such as phalloidin, which is used to provide tight and selective binding to actin in cells (for further details on the functionalization see the Methods section). Two centrifugation steps were carried out in order to fully purify the AuNRs. Spectral characterization of the supernatant and pellet confirmed the removal of excess dye.
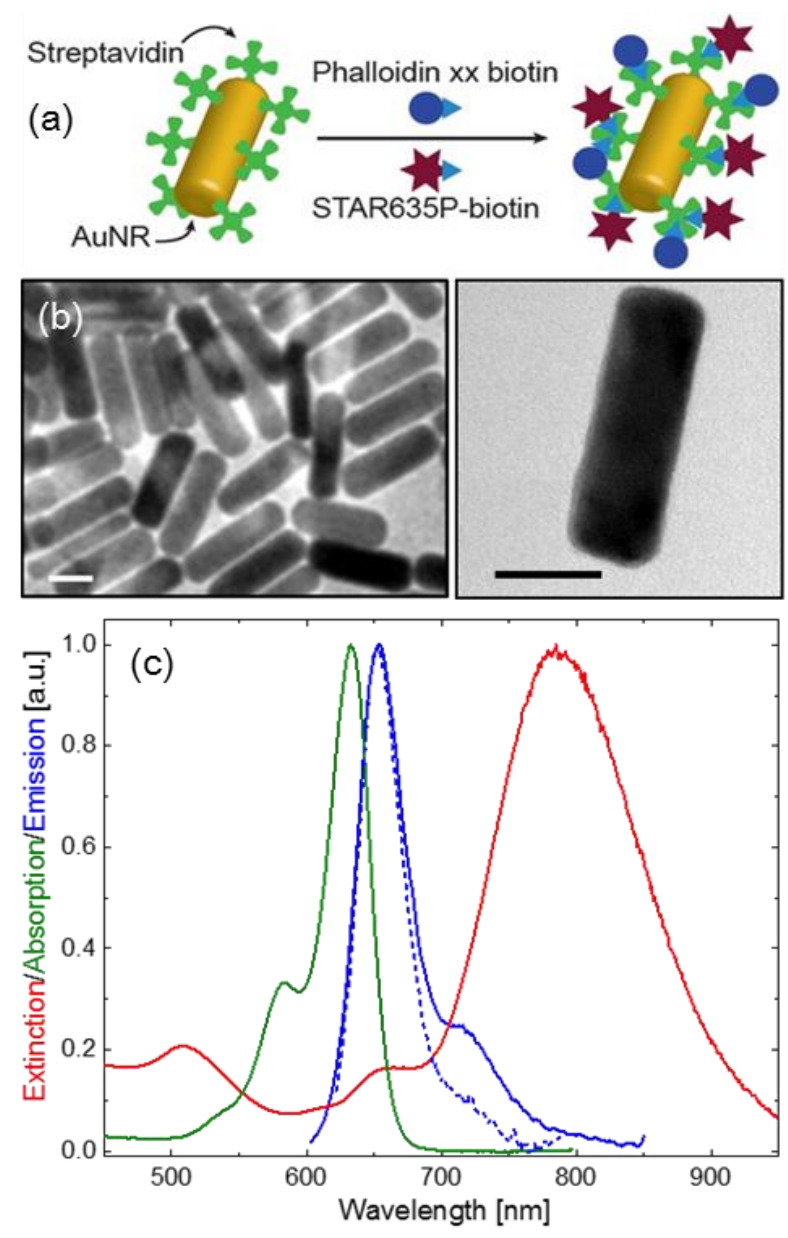

Figure 1: (a) Scheme of the functionalization procedure employed to synthesize the plasmonicfluorescent-probes. Streptavidin coated AuNRs, featuring approximately 15 streptavidin proteins per nanorod, were incubated with biotinylated phalloidin $(20 \mu \mathrm{M}$ in $\mathrm{MeOH}, 50 \mu \mathrm{L})$ and biotinylated STAR $635 P$ dye $(0.1 \mathrm{mg} / \mathrm{mL}$ in DMSO, $14 \mu \mathrm{L})$. After 3 hours the mixture was centrifuged twice, before resuspending the pellet in $\mathrm{H}_{2} \mathrm{O}$. (b) Representative TEM images of the AuNRs. Scale bars: $10 \mathrm{~nm}$. (c) Optical characterization of the AuNRs. Green (blue) line is the absorption (emission) spectrum of the dye (STAR 635P). The blue dashed line shows the emission of the dye once bound to the AuNRs (excited at $600 \mathrm{~nm}$ ). The red line is the extinction spectrum of a STAR 635P-AuNRs thin film, measured in ProLong Gold media and showing the transversal and longitudinal plasmon resonances at $520 \mathrm{~nm}$ and $780 \mathrm{~nm}$, respectively.

The average size of our AuNRs (26 $\pm 4 \mathrm{~nm}$ length and $8 \pm 1 \mathrm{~nm}$ width) was determined by TEM ( $N>100$ particles - see Figure S2 for distributions). Figure $1(\mathrm{~b})$ shows representative TEM images of the post-functionalized AuNRs. We estimate the total size of the probe - metal particle and capping layer to be $\sim 36 \times 18 \mathrm{~nm}$. The extinction spectrum of the $\sim 3.25$ aspect ratio AuNRs measured in ProLong Gold 
media shows LSPRs centered at $520 \mathrm{~nm}$ (transversal mode) and $780 \mathrm{~nm}$ (longitudinal mode) - see red line in Figure 1(c); in agreement with calculations (see Figure S3). We also present in Figure 1(c) the absorption (green line) and emission spectrum (dashed blue line; excited at $600 \mathrm{~nm}$ ) of the STAR 635P conjugated to the AuNRs. The corresponding emission spectrum of the free STAR 635P dye is also shown (solid blue line). The fluorescence lifetime was found to be the same for the AuNR and the free dye (see Figure S4) and the emission spectra are similar apart from an apparent decrease at the red edge that we attribute to emitted photons being absorbed by the AuNRs at those wavelengths.

In order to characterize the electromagnetic response of the employed AuNRs, their extinction cross section was calculated by means of finite element method simulations (see Methods section for more details). Figure 2(a) presents the calculated extinction cross section of an AuNR (under plane wave illumination), showing the longitudinal LSPR at $780 \mathrm{~nm}$ and the transverse LSPR at $520 \mathrm{~nm}$, in close agreement with the measured spectrum (see Figure 1(c)). The inset panel (Figure 2(a)) confirms that the longitudinal LSPR supported by the AuNRs at $780 \mathrm{~nm}$ corresponds to a dipolar plasmon resonance excited by the electric field component of the incident wave parallel to the particle's long axis. This panel exemplifies the large field enhancement at the LSPR, reaching values over 35 close to the rod's tips surface, where the field enhancement is at its maximum. The spatial region of a few $\mathrm{nm}$ away from the metal is known to cause quenching of the dye through non-radiative decay channels in the metal. As discussed above, by linking the dyes to the AuNRs surface with the biotin/streptavidin conjugation, they are kept at an average distance of $5 \mathrm{~nm}$ from the metal, thus, avoiding the quenching of the plasmonicfluorescent nanoprobes. ${ }^{30,31}$ At this distance, the still-appreciable field enhancement produced by the AuNR (see Figure 2(b)) causes and enhancement of the depletion intensity experienced by the fluorophores.
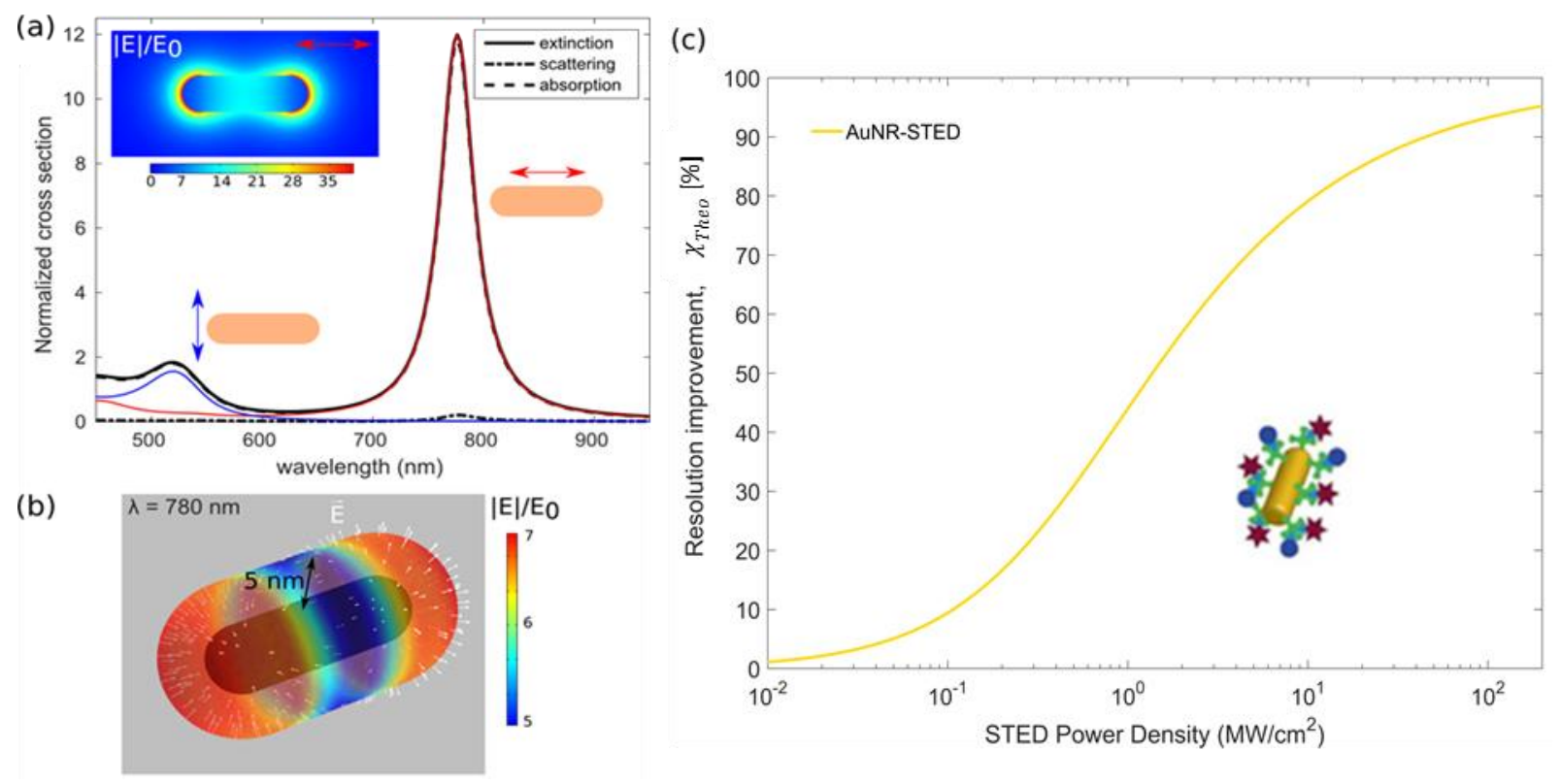
Figure 2: (a) Simulated spectrum of AuNRs (26x8 nm) in oil ( $n=1.47$, to reproduce the experimental conditions). The solid lines show the extinction cross section for incident light polarized along the rod's long axis (red), short axis (blue) and the corresponding for circularly polarized light (black). The absorption and scattering contributions to the extinction are given with dashed and dotted-dashed lines, respectively. Inset panel: on-resonance electric field enhancement $(\lambda=780 \mathrm{~nm})$ for under excitation with the $\boldsymbol{E}$ component along the rod's axis (note the wave's power corresponds to a circularly polarized plane wave). (b) Field enhancement and vector field on a surface $5 \mathrm{~nm}$ away from the rod's surface, the average value of the fluorophore distance to the rod. (c) Orange line shows the calculated resolution improvement (\%) curve $\chi_{\text {Theo }}$ as a function of the depletion (STED) average laser power densities using our fluorescent-AuNRs. The fluorescent dye STAR 635P was assumed for the calculations.

To demonstrate the potential of the AuNRs for NP-STED, we functionalized a glass cover-slip to attach the AuNRs to the surface (see Methods for further details) and compared the resolution improvement relative to confocal microscopy for the AuNR and for $20 \mathrm{~nm}$ diameter crimson red beads as a function of the power of the depletion-beam. Assuming that the depletion beam pulse length, $\tau_{S T E D}$, is much smaller than the fluorescence lifetime of the fluorophore, $\tau_{S 1}$, the resolution achievable with pulsed STED depends on the wavelength, $\lambda$, the numerical aperture (NA) of the objective, the peak intensity of the depletion beam, $I_{S T E D}$, and the saturation intensity of the fluorophore, $I_{\text {sat }}$, as given by: ${ }^{16,32}$

$$
F W H M_{S T E D}=\frac{F W H M_{0}}{\sqrt{(1+\emptyset)}}
$$

where

$$
F W H M_{0}=\frac{0.51 \lambda}{N A}
$$

and

$$
\emptyset=\frac{I_{S T E D} \tau_{S T E D}}{I_{S a t} \tau_{S 1}}
$$

Hence, we can write the STED resolution improvement with respect to confocal microscopy as $\Gamma_{\text {res }}=$ $\sqrt{1+\emptyset}$. In the case of NP-STED, we can introduce an extra factor describing the spatially-averaged improvement in resolution due to the nanoparticle. The resolution improvement factor of NP-STED nanoscopy ${ }^{16}$ can, therefore, be written as:

$$
\Gamma_{\text {res }}=\sqrt{1+\Gamma_{p} \emptyset}
$$

where

$$
\Gamma_{p}=\frac{\Gamma_{\mathrm{I}}}{\gamma}
$$


Here, $\Gamma_{I}$ is the local increase of the depletion intensity at the fluorophores location due to the electromagnetic field enhancement (at the STED wavelength), and $\gamma$ is the enhancement of the decay rate of the dye, which is modified due to the Purcell effect (at the emission wavelength). An increase in the fluorescent decay rate is detrimental as it reduces the total amount of collected photons. For this reason, the metal NPs were designed to be out of resonance at the dye emission wavelength, such that $\gamma \approx 1$, which was confirmed by the lifetime measurements of the sample (see Figure S4). ${ }^{33}$

The resolution improvement of the NP-STED system is, therefore, estimated by means of the electromagnetic intensity enhancement, $\Gamma_{\mathrm{I}}$. Such enhancement factor can be calculated for the AuNRs under plane wave illumination. Indeed, it has been shown ${ }^{34}$ that such an estimate provides a crude but valuable and easy-to-implement alternative for exact numerical simulations, such as those performed in Ref 15 . Those simulations have also shown that the doughnut's beam shape is not significantly affected by the presence of the nanoparticles. ${ }^{15}$ On the other hand, since the fluorophores are randomly positioned over the rod at $\sim 5 \mathrm{~nm}$ from its surface and with randomly oriented dipole moments, the averaged value of the local enhancement $\bar{\Gamma}_{\mathrm{I}}$, has to be considered. As shown in Figure 2(b), the field enhancement at that distance from the rod ranges from 5 to 7 . Averaging the local field intensity over the fluorophore's positions and orientations yields an intensity enhancement factor $\bar{\Gamma}_{\mathrm{I}} \approx 8.5$ (see Methods). Yet, the effective enhancement is reduced because of the lower emission cross section of the hybrid STAR 635P-AuNR emitter with respect to the free dye, see Fig. 1(c), which gives rise to a higher saturation level. With this, we characterize the resolution improvement factor in percent via the expression. ${ }^{17}$

$$
\chi_{\text {Theo }}=100 \cdot\left(1-\frac{1}{\Gamma_{\text {res }}}\right)
$$

This calculated resolution improvement curve $\left(\chi_{\text {Theo }}\right)$ is shown in Figure 2(c) for the STAR 635P-AuNRs. This can be directly compared to the measured resolution improvement:

$$
\chi_{E x p}=100 \cdot\left(1-\frac{F W H M_{S T E D}}{F H M W_{\text {conf }}}\right)
$$

By comparing the FWHM of the bright spots (i.e. single fluorescent-AuNR or single fluorescence bead) observed from the AuNRs under confocal or STED imaging, it is possible to estimate this resolution improvement (\%) factor, $\chi_{\text {Exp }}$, experimentally for a given STED depletion-power, as exemplified in Figure 3(b) (see Figures S6 and S7 for more examples). Figure 3(a) shows the experimental resolution improvement, $\chi_{E x p}$, for fluorescent-AuNRs (orange crosses) and for the non-plasmonic probes (20 nm crimson fluorescent beads - green crosses - see experimental methods for details). This was determined for selected AuNR/beads across the sample as a function of the power of the depletion beam; see examples in Figures 3(b) and 3(c). Normalized intensity profiles, for better comparison, are presented in Figure S8. 

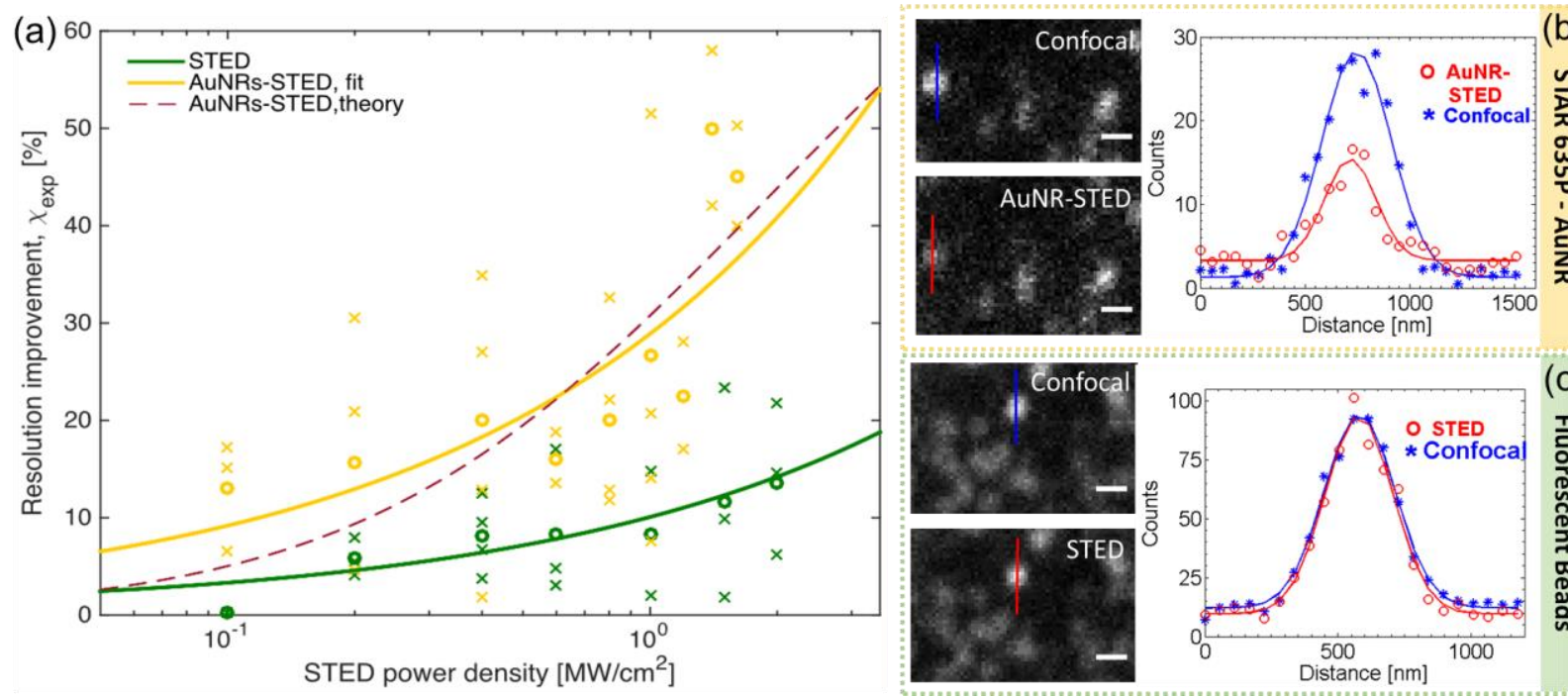

(c)

Figure 3: (a) Orange/green crosses are experimental measurements for fluorescent-AuNRs/fluorescent beads at different depletion average power densities and orange/green open circles are the average resolution improvement for AuNRs-STED/fluorescent beads-STED. Lines are fits for the average behaviour. The dashed red line shows the resolution improvement based on the calculated field enhancement and emission cross section of the hybrid STAR 635P-AuNR emitter .(b) Fluorescent-AuNR confocal (top) and NP-STED (bottom) images and the corresponding fittings showing the resolution improvement for the selected rod (marked with lines in the images) using depletion laser intensities of $0.2 \mathrm{MW} / \mathrm{cm}^{2}$. (c) Bottom panel: under the same conditions, using $20 \mathrm{~nm}$ fluorescent beads, there is little improvement in resolution for such a low depletion power $\left(0.2 \mathrm{MW} / \mathrm{cm}^{2}\right)$. ProLong Gold is used as indexmatching media for AuNRs and TDE (97\%) for the fluorescent beads sample. Scale bars in images correspond to $500 \mathrm{~nm}$.

Although there is a significant dispersion in the measurements for both systems (which in the case of AuNRs may arise from clustering of the NPs, from a finite AuNRs size-distribution or from thermallyinduced motion of the AuNR as discussed below), there is a clear trend indicated by the average values found for each depletion power (open circles in Figure 3(a)). For depletion-powers as low as 1.5 $\mathrm{MW} / \mathrm{cm}^{2}$, we observed a resolution improvement of up to $\sim 50 \%$ for the AuNRs with respect to the confocal resolution - in very good agreement with the numerical estimates of Figure 2(c) - while for the fluorescent beads this value is below $10 \%$ under the same experimental conditions. This implies an upper limit improvement of $44 \%$ for AuNR-STED compared to dye-based STED at this depletion power. Equivalently, when using the AuNRs, a given resolution improvement is attained by about 10 times lower intensity compared with the control measurement. In fact, since crimson beads have a lower saturation intensity than STAR 635P (see Methods section), a comparison of the AuNRs against beads labelled with STAR635P would be expected to show an even larger improvement in resolution.

As well as these plasmonic probes for STED being 2000 times smaller in volume than the previously used silica@Au particles, ${ }^{17}$ the average near field intensity experienced by the fluorophores in the case of the AuNRs is 2 times larger at the depletion wavelength (see Methods section and Figure S5). The 
amount of gold in each AuNR is also greatly reduced (1000 times) compared to the Au shell of previously used particles. This reduces the gold luminescence below our detection limit and is not apparent in the emission decay profiles (see SI). Thus, we do not need to apply time-gated detection to prevent the spurious luminescence compromising the STED performance, as was necessary for the core@shell nanoparticles ${ }^{17}$ and which decreased the detected fluorescence signals. Unfortunately, while the shape and small size of the chosen NPs provides a large electric field enhancement and the possibility to incorporate them into cells (as shown next), this is accompanied by an increase in the absorption of the metal. In practice, this leads to heating and thermally induced movement of the AuNRs that becomes significantly challenging for STED nanoscopy with depletion powers over $\sim 2 \mathrm{MW} / \mathrm{cm}^{2}$ (see further discussion in the SI). This clearly presents a practical challenge for NP-STED, as further discussed in the conclusions.

Finally, we incorporated fluorescently labelled AuNRs into adult neural stem cells in order to perform super-resolution imaging inside the cell (see Methods for details). Figure 4 shows an initial study, where the resolution improvement for NP-STED can be clearly seen across the image for depletion powers as low as $0.5 \mathrm{MW} / \mathrm{cm}^{2}$. However, it is important to take into account the anisotropic nature of these AuNRs that would be bound to actin and the potential impact on the resolution improvement of out-of-plane orientations. In order to investigate this possibility, we performed calculations on the "effective" point spread function (PSF) of the depletion beam and the expected emission PSF, depending on the 3D orientation of the AuNRs (see Figures S10-S12). Our calculations show that the degree of improvement in resolution can vary depending on the specific orientation of the AuNRs. This last fact can account for the brighter/dimmer spots shown in Figure 4(c) compared to 4(b). Although further work is required to improve the labeling of relevant intracellular structures (see Figure S13), this proof of concept experiment nevertheless indicates the potential for lower power STED nanoscopy of biological samples. $^{22}$

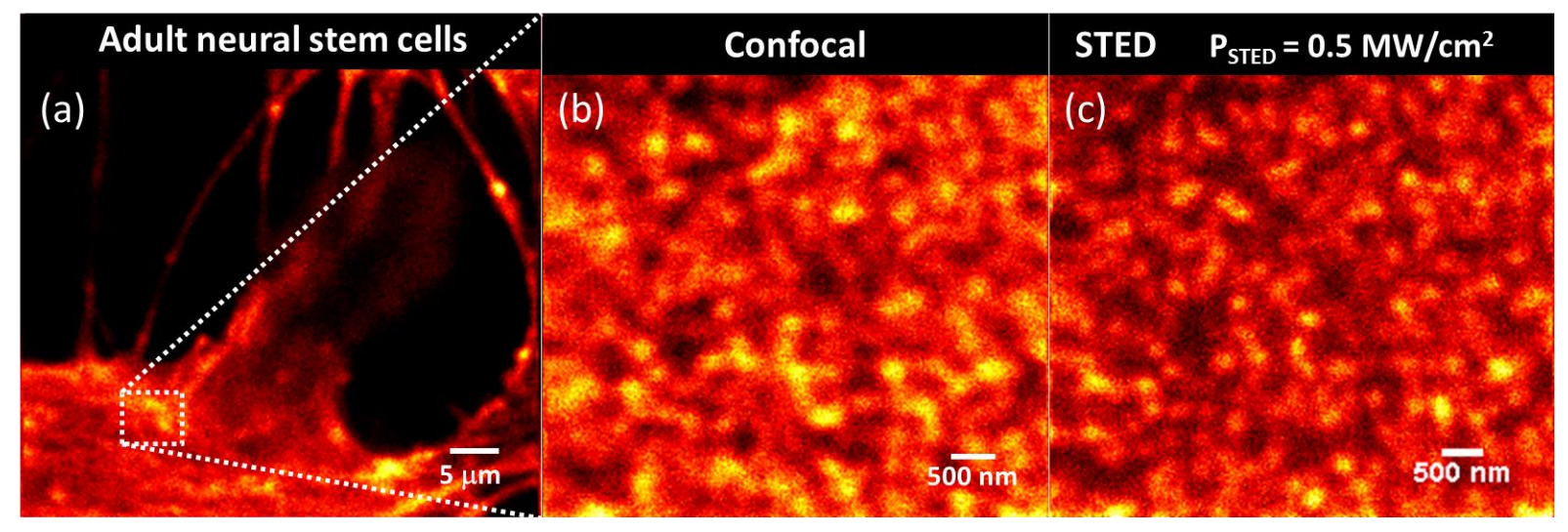

Figure 4: a) AuNRs incorporation into adult neural stem cells. b) Confocal versus c) NP-STED images inside the cell (zoom from the marked region in a). Depletion-beam power for the NP-STED case was set at $0.5 \mathrm{MW} / \mathrm{cm}^{2}$.

\section{Conclusions:}


While many different types of nanoparticles (made out of, e.g. silica, carbon, polymers, quantum dots, etc.) have been utilised as probes for fluorescence imaging, ${ }^{35}$ super-resolved bioimaging with nanoparticles has only recently been accomplished - using quantum dots. ${ }^{14}$ In this report, we have shown the possibility to extend the NP-STED concept to significantly smaller nanometric plasmonic probes that we have shown are able to operate at low powers and can be incorporated into cells. Employing the near-field enhancement properties of fluorescently labelled $26 \times 8 \mathrm{~nm}$ AuNRs, we achieve a resolution improvement of $50 \%$ compared to $10 \%$ for STED nanoscopy of fluorescent beads with the same depletion power. We further note that the smaller size of these AuNRs compared to the core@shell NPs ${ }^{17}$ eliminates the issue of unwanted background light from gold luminescence. This demonstrates the potential of plasmon-assisted STED to realize super-resolution at lower depletion powers. This could benefit rapid parallelized STED microscopy by reducing the total power required to realise the multiple-doughnut approach. ${ }^{12,13}$ Further advantages of performing STED at lower depletion powers (NP-STED) could include reducing unwanted autofluorescence background in cells excited by the depletion beam and reducing phototoxicity.

Currently, the main challenge for NP-STED for bioimaging is the heating of the NPs and surrounding medium upon illumination, which has limited the resolution achieved with the AuNRs. In addition, heating can lead to thermally-induced motion of the NPs and ultimately their destruction at higher depletion powers. ${ }^{17}$ It may be possible to mitigate these issues to some extent by optimizing the duration and repetition rate of the STED pulses and taking advantage of the rapid equilibration of the NPs temperature, which we calculate to be $\sim 6 \mathrm{~ns}$ (see figure S9). ${ }^{36}$ For instance, by increasing the duration of the pulses (i.e. 1 ns) while maintaining the total pulse energy, it is possible to reduce the peak temperature rise by over $50 \%$ (see Figure S9) - although time-gated detection may then be necessary to optimize the STED nanoscopy resolution. Another possibility would be to reduce the depletion intensity required to deplete the dye by working at shorter wavelengths. ${ }^{37,38}$ Specifically, designing anisotropic NPs with a LSPR centered at $\sim 750 \mathrm{~nm}$, where the cross-section for stimulated emission depletion of STAR635P is approximately 3 times higher than at $780 \mathrm{~nm}$, could reduce the intensity required to achieve a given STED resolution by the same factor of $\sim 3$, thus, further reducing the heat generated. Engineering smaller plasmonic particles could also reduce the amount of heat generated, however, the field-enhancements obtained with such particles would be lower. This could be compensated by locating the emitters closer to the metal surface, at the cost of reducing their quantum yield and fluorescence lifetime. However, the lower photon output could be compensated by a concomitant reduction of photobleaching. ${ }^{47,48,49}$

Finally, as a further development of the NP-STED concept, we envision the possibility of combining plasmonic particles with quantum dots (as fluorophores) in order to have a robust probe in terms of photobleaching ${ }^{14}$ with the plasmon modes enhancing the depletion of the emission, ${ }^{39,40}$ thus, requiring less power for STED nanoscopy.

\section{Methods:}


Synthesis and Functionalization of AuNRs: Streptavidin coated AuNRs (Nanopartz, $150 \mu \mathrm{L}$ ), featuring approximately 15 streptavidin proteins per nanorod, were incubated with phalloidin-xx- biotin ( $20 \mu \mathrm{M}$ in $\mathrm{MeOH}, 50 \mu \mathrm{L}$ ) and biotinylated STAR 635P (Abberior) dye $(0.1 \mathrm{mg} / \mathrm{mL}$ in DMSO, $14 \mu \mathrm{L}$ ). After 3 hours the mixture was centrifugally washed at $9660 \times g$ twice before resuspending the pellet in $1 \mathrm{~mL}$ of $\mathrm{H}_{2} \mathrm{O}$ (18 $\mathrm{M} \Omega$, ultrapure). Calculated molar quantities: Streptavidin $-2.28 \times 10^{-10}$ moles; phalloidin-xx-biotin $-1 \mathrm{x}$ $10^{-9}$ moles; STAR 635P - $1.1 \times 10^{-9}$ moles. We estimate a labelling ratio of 7 fluorophores per nanorod. A control sample was made up in the same way without the dye, and with $150 \mu \mathrm{L}$ of phalloidin-xx-biotin to ensure correct rejection from the excitation and STED lasers (no fluorophores) and discard interference (i.e. scattering) from the metallic nanoparticles. Absorption and emission spectra were measured in solution using water as solvent. $I_{\text {sat }} \sim 15 \mathrm{MW} / \mathrm{cm}^{2}$ was estimated for STAR $635 \mathrm{P}$ from its lifetime, $\tau$, and extinction coefficient, $\sigma$, given by the data sheet [http://www.sigmaaldrich.com/lifescience/cell-biology/detection/abberior-dyes.html], as $I_{\text {sat }}=k \cdot h \cdot c /\left(\lambda_{S T E D} \sigma\right)$, where $k$ is the spontaneous decay rate, $k=1 / \tau$, and $\sigma$ is the extinction coefficient at the STED wavelength.

Fixation of AuNRs to the substrate: AuNRs were fixed on glass substrates by soaking the substrates in PBS 3 times, 10 minutes each. After that, a mixture of $1 \mathrm{mg} / \mathrm{ml}$ biotinylated BSA and $0.1 \mathrm{mg} / \mathrm{ml} \mathrm{BSA}$ was left in contact with the substrates for 3 hours followed by 3 washing steps with PBS. AuNRs were diluted 50 times in PBS (final OD close to 1) and then put in contact with substrate for 2 hours followed by washing 3 times with PBS. Finally, biotinylated STAR 635P (1 mM) was used to label the fixed rods in the substrate and then washed two times with PBS.

Preparation of fluorescent bead sample: $20 \mathrm{~nm}$ diameter fluorescent beads (Crimson beads, Invitrogen, Carlsbad, CA, US) with excitation/emission maxima at 625/645 nm were diluted 1:1000 in MilliQ and applied to Poly-L-lysine coated coverslips. They were mounted in TDE (97\%) in order to match the refractive index of 1.52 for the immersion oil. $I_{\text {sat }} \sim 2 \mathrm{MW} / \mathrm{cm}^{2}$, was estimated for crimson beads from Ref 41.

Scattering of gold nanoparticles: The electromagnetic response of the nanoparticles is characterized by the extinction cross section under plane wave incidence. Extinction is given by the sum of scattering and absorption, which were calculated using the finite element method [Comsol Multiphysics, https://www.comsol.com/comsol-multiphysics]. The scattering cross section is obtained from the power scattered by the nanorod and the absorption cross section from the electromagnetic energy absorbed by the particle. The calculation is performed for two different incident polarizations (electric field parallel to the long or short axis of the rod), and circular polarization is then considered. The results shown in Figure 2(a) correspond to cross sections normalized to the illuminated area of the particle. The permittivity of gold, $\varepsilon_{A u}(\omega)$, was taken from the data given in reference 42 .

Plasmonic enhancement factor of STED resolution: As discussed in the main text, the NP-STED resolution improvement is given by $\Gamma_{\text {res }} \approx \sqrt{1+\Gamma_{P} \phi}$, where $\Gamma_{P} \approx \bar{\Gamma}_{I}$. In order to calculate $\bar{\Gamma}_{I}$ we perform a series of averages of the dipole orientations of the AuNR. We start from a simulation with a linearly polarized plane wave, since only the electric field component of the circularly polarized beam that lies along the rod's long axis excites the longitudinal LSPR (note that we take the appropriate normalization factor into account and that all the field enhancement values given in this paper 
correspond to a circularly polarized wave). Next, we average the intensity enhancement at the fluorophore position, $\Gamma_{I}(\boldsymbol{r})=\left|\boldsymbol{\mu} \cdot \boldsymbol{E}_{S P}(\boldsymbol{r})\right|^{2}$, over random dipole orientations ( $\boldsymbol{\mu}$ is the unit dipole moment in the direction of the fluorophore's dipole and $\boldsymbol{E}_{S P}(\boldsymbol{r})$ the near field enhancement at the LSPR). Finally, in order to take into account the inhomogeneous field distribution provided by the AuNRs we average over random positions over the AuNR, $\bar{\Gamma}_{I}=1 / A \int \mathrm{d} \boldsymbol{A} \cdot \Gamma_{I}(\boldsymbol{r})$, with $A$ being the area of the surface $5 \mathrm{~nm}$ away from the AuNR, as depicted in Fig. 2(b). After performing all the averages, we obtain a plasmonic intensity enhancement factor of 8.5, which corresponds to an average field enhancement for the fluorophores of 3 . Note that in case not all the rods lie on the plane, as could potentially be for the bioimaging example shown in Figure 4, the averaged plasmonic intensity enhancement factor would change. This is discussed further in the SI.

STED microscopy set-up: The STED-FLIM microscope employed in this study is a development of that described previously ${ }^{43,44}$ utilising a single $80 \mathrm{MHz}$ repetition rate mode-locked Ti:Sapphire laser (SpectraPhysics, Mai Tai HP) tuned to $780 \mathrm{~nm}$ to provide the depletion beam and to generate a supercontinuum in a microstructured fibre from which the excitation pulses were selected using a bandpass filter $(628 / 40 \mathrm{~nm})$. Further details on the experimental set-up are presented in the SI.

STED microscopy: STED images were taken by first acquiring a confocal image by scanning 10 frames and adding them together to give a final image. The acquisition time was $0.41 \mathrm{~s}$ for each $64 \times 64$ frame, where the pixel size was $55.96 \mathrm{~nm}$. Once the confocal image was acquired, the depletion beam was switched on and a STED image was acquired by scanning and summing 10 frames. The depletion laser pulses were set to arrive $10 \mathrm{ps}$ after the excitation pulses. The average power of the depletion beam was measured before the tube lens of the microscope and the STED pulse intensity $\left(I_{S T E D}\right)$ was calculated using $\mathrm{I}_{S T E D}=k \frac{P_{S T E D}}{A_{S T E D}}$, where $k=0.3, A_{S T E D}$ is the STED focal area and $P_{S T E D}$ the corresponding peak power ${ }^{45}$ obtained by dividing the pulse energy by $\tau_{S T E D}$.

FLIM microscopy: FLIM (Fluorescence Lifetime IMaging) data was acquired in confocal imaging mode by accumulating five $64 \times 64$ frames, where the pixel size was $55.96 \mathrm{~nm}$ and the acquisition time was $0.41 \mathrm{~s}$ for each frame. Fluorescence intensity images were analyzed using Fiji and MatLab 2013a (The Mathworks, Inc, Natick, Massachusetts, US) and FLIM data was analyzed using our in-house open source tool, FLIMfit. ${ }^{46}$

Cell stain, fixation and measurements: Adult neural stem cells were treated for 15 minutes with $4 \%$ paraformaldehyde (PFA) in PBS buffer and permeabilised for 10 minutes with $0.5 \%$ triton-x100 detergent in PBS at room temperature. One litre of PBS buffer was made out of: $8 \mathrm{~g} \mathrm{NaCl}, 0.2 \mathrm{~g} \mathrm{KCl}, 0.2 \mathrm{~g}$ $\mathrm{KH}_{2} \mathrm{PO}_{4}, 1.15 \mathrm{~g} \mathrm{Na}_{2} \mathrm{HPO}_{4}$. After that the cells were blocked for 30 minutes with $3 \%$ bovine serum albumin (BSA) in PBS at room temperature. Finally, cell's staining was carried out with a dilution of AuNRsAtto647-phalloidin probes (resonant at $820 \mathrm{~nm}$ in ProLong Gold media) in a solution of 3\% BSA in PBS (in order to reach an optical density of $\sim 1$ ) for 1 hour at room temperature, washed 3 times for 10 minutes with PBS and mounted in ProLong Gold anti-fade reagent. Depletion wavelength was set to $820 \mathrm{~nm}$ in this case. 


\section{Supplementary Information:}

The Supporting Information is available free of charge on the ACS Publications website www.pubs.acs.org

STED set-up, Nanorods size-distribution, LSPR dependence on AuNRs aspect ratio and embedding medium, Lifetime measurements, Nanoshells vs. nanorods, Additional NP-STED images, Normalized intensity profiles, Photothermal effects, AuNRs-STED anisotropy, Actin labelling with AuNRs.

\section{Acknowledgements:}

This project was supported by the MRC Next Generation Optical Microscopy Initiative, (MR/K015834/1), EPSRC Reactive Plasmonics project EP/M013812/1, the Royal Society and the Leverhulme Trust. EC and PAH are supported by Marie-Sklodowska Curie fellowships. WP is supported by an EPSRC Doctoral Prize Fellowship (EP/M506448/1). HS acknowledges a CASE Ph.D studentship from the UK Engineering and Physical Sciences Research Council (EPSRC) and the Diamond Trading Company. YS acknowledges the support from the People Programme (Marie Curie Actions) of the European Union's Seventh Framework Programme (FP7/2007-2013) under REA grant agreement no.333790, as well as the support of the Alumni program of the International Newton Fellowship program of the Royal Society and of the Israeli national nanotechnology Initiative. SAM acknowledgers the Lee-Lucas Chair in Physics. We want to acknowledge Dr. Yannick Sonnefraud for initial discussions on this project.

\section{References:}

${ }^{1}$ Hell, S. W.; Wichmann, J. Breaking the Diffraction Resolution Limit by Stimulated Emission: StimulatedEmission-Depletion Fluorescence Microscopy. Opt. Lett. 1994, 19, 780-782.

2 Klar, T. A.; Jakobs, S.; Dyba, M.; Egner, A.; Hell, S. W. Fluorescence Microscopy with Diffraction Resolution Barrier Broken by Stimulated Emission. PNAS 2000, 97, 8206-8210.

${ }^{3}$ Hell, S. W. Toward Fluorescence Nanoscopy. Nat. Biotech. 2003, 21, 1347-1355.

${ }^{4}$ Hofmann, M.; Eggeling, C.; Jakobs, S.; Hell, S. W. Breaking the diffraction barrier in fluorescence microscopy at low light intensities by using reversibly photoswitchable proteins. PNAS 2005, 102, 1756517569.

${ }^{5}$ Betzig, E.; Patterson, G. H.; Sougrat, R.; Lindwasser, O. W.; Olenych, S.; Bonifacino, J. S.; Davidson, M. W.; Lippincott-Schwartz, J.; Hess, H. F. Imaging Intracellular Fluorescent Proteins at Nanometer Resolution. Science 2006, 313, 1642-1645.

6 Hess, S. T.; Girirajan, T. P. K.; Mason, M. D. Ultra-High Resolution Imaging by Fluorescence Photoactivation Localization Microscopy. Biophys. J., 91, 4258-4272. 
7 Rust, M. J.; Bates, M.; Zhuang, X. Sub-diffraction-limit Imaging by Stochastic Optical Reconstruction Microscopy (STORM). Nat. Methods 2006, 3, 793-796.

8 Orrit, M. Nobel Prize in Chemistry: Celebrating Optical Nanoscopy. Nat. Photonics 2014, 8, 887-888.

${ }^{9}$ Willig, K. I.; Kellner, R. R.; Medda, R.; Hein, B.; Jakobs, S.; Hell, S. W. Nanoscale Resolution in GFPBased Microscopy. Nat. Methods 2006, 3, 721-723.

10 Berning, S.; Willig, K. I.; Steffens, H.; Dibaj, P.; Hell, S. W. Nanoscopy in a Living Mouse Brain. Science 2012, 335, 551-551.

11 Rittweger, E.; Han, K. Y.; Irvine, S. E.; Eggeling, C.; Hell, S. W. STED Microscopy Reveals Crystal Colour Centres with Nanometric Resolution. Nat. Photonics 2009, 3, 144-147.

12 Bingen, P.; Reuss, M.; Engelhardt, J.; Hell, S. W. Parallelized STED Fluorescence Nanoscopy. Opt. Express 2011, 19, 23716-23726.

13 Yang, B.; Przybilla, F.; Mestre, M.; Trebbia, J.-B.; Lounis, B. Large Parallelization of STED Nanoscopy Using Optical Lattices. Opt. Express 2014, 22, 5581-5589.

14 Hanne, J.; Falk, H. J.; Gorlitz, F.; Hoyer, P.; Engelhardt, J.; Sahl, S. J.; Hell, S. W. STED Nanoscopy with Fluorescent Quantum Dots. Nat. Commun. 2015, 6.

15 Sivan, Y.; Sonnefraud, Y.; Kéna-Cohen, S.; Pendry, J. B.; Maier, S. A. Nanoparticle-Assisted Stimulated-Emission-Depletion Nanoscopy. ACS Nano 2012, 6, 5291-5296.

16 Sivan, Y. Performance Improvement in Nanoparticle-Assisted Stimulated-Emission-Depletion Nanoscopy. Appl. Phys. Lett. 2012, 101, 021111.

17 Sonnefraud, Y.; Sinclair, H. G.; Sivan, Y.; Foreman, M. R.; Dunsby, C. W.; Neil, M. A. A.; French, P. M.; Maier, S. A. Experimental Proof of Concept of Nanoparticle-Assisted STED. Nano Letters 2014, 14, 44494453.

18 Giannini, V.; Fernández-Domínguez, A. I.; Heck, S. C.; Maier, S. A. Plasmonic Nanoantennas: Fundamentals and Their Use in Controlling the Radiative Properties of Nanoemitters. Chem. Rev. 2011, $111,3888-3912$.

19 Halas, N. J.; Lal, S.; Chang, W.-S.; Link, S.; Nordlander, P. Plasmons in Strongly Coupled Metallic Nanostructures. Chem. Rev. 2011, 111, 3913-3961.

20 Wu, H.-Y.; Huang, Y.-T.; Shen, P.-T.; Lee, H.; Oketani, R.; Yonemaru, Y.; Yamanaka, M.; Shoji, S.; Lin, K.-H.; Chang, C.-W.; Kawata, S.; Fujita, K.; Chu, S.-W. Ultrasmall All-Optical Plasmonic Switch and its Application to Superresolution Imaging. Sci. Rep. 2016, 6, 24293.

${ }^{21}$ Chu, S.-W.; Su, T.-Y.; Oketani, R.; Huang, Y.-T.; Wu, H.-Y.; Yonemaru, Y.; Yamanaka, M.; Lee, H.; Zhuo, G.-Y.; Lee, M.-Y.; Kawata, S.; Fujita, K. Measurement of a Saturated Emission of Optical Radiation from Gold Nanoparticles: Application to an Ultrahigh Resolution Microscope. Phys. Rev. Lett. 2014, 112, 017402. 
${ }^{22}$ Lee, S. A.; Ponjavic, A.; Siv, C.; Lee, S. F.; Biteen, J. S. Nanoscopic Cellular Imaging: Confinement Broadens Understanding. ACS Nano 2016, 10, 8143-8153.

${ }^{23}$ Blythe, K. L.; Titus, E. J.; Willets, K. A. Effects of Tuning Fluorophore Density, Identity, and Spacing on Reconstructed Images in Super-Resolution Imaging of Fluorophore-Labeled Gold Nanorods. J. Phys. Chem.C 2015, 119, 28099-28110.

24 Albanese, A.; Tang, P. S.; Chan, W. C. W. The Effect of Nanoparticle Size, Shape, and Surface Chemistry on Biological Systems. Annu. Rev. Biomed. Eng. 2012, 14, 1-16.

${ }^{25} \mathrm{Ni}$, M.; Zhuo, S.; So, P. T. C.; Yu, H. Fluorescent Probes for Nanoscopy: Four Categories and Multiple Possibilities. J. Biophotonics 2016, n/a-n/a.

${ }^{26}$ Maier, S. A.: Plasmonics: Fundamentals and Applications; 1 ed.; Springer US, 2007.

${ }^{27}$ Eustis, S.; El-Sayed, M. A. Why Gold Nanoparticles are More Precious than Pretty Gold: Noble Metal Surface Plasmon Resonance and its Enhancement of the Radiative and Nonradiative Properties of Nanocrystals of Different Shapes. Chem. Soc. Rev. 2006, 35, 209-217.

28 Link, S.; Mohamed, M. B.; El-Sayed, M. A. Simulation of the Optical Absorption Spectra of Gold Nanorods as a Function of Their Aspect Ratio and the Effect of the Medium Dielectric Constant. J. Phys. Chem. B 1999, 103, 3073-3077.

29 Balzarotti, F.; Stefani, F. D. Plasmonics Meets Far-Field Optical Nanoscopy. ACS Nano 2012, 6, 45804584.

30 Anger, P.; Bharadwaj, P.; Novotny, L. Enhancement and Quenching of Single-Molecule Fluorescence. Phys. Rev. Lett. 2006, 96, 113002.

31 Darst, S. A.; Ahlers, M.; Meller, P. H.; Kubalek, E. W.; Blankenburg, R.; Ribi, H. O.; Ringsdorf, H.; Kornberg, R. D. Two-dimensional Crystals of Streptavidin on Biotinylated Lipid Layers and their Interactions with Biotinylated Macromolecules. Biophys. J. 1991, 59, 387-396.

32 Leutenegger, M.; Eggeling, C.; Hell, S. W. Analytical Description of STED Microscopy Performance. Opt. Express 2010, 18, 26417-26429.

${ }^{33}$ Fixler, D.; Nayhoz, T.; Ray, K. Diffusion Reflection and Fluorescence Lifetime Imaging Microscopy Study of Fluorophore-Conjugated Gold Nanoparticles or Nanorods in Solid Phantoms. ACS Photonics 2014, 1, 900-905.

34 a) Foreman, M. R.; Sivan, Y.; Maier, S. A.; Török, P. Independence of Plasmonic Near-Field Enhancements to Illumination Beam Profile. Phys. Rev. B 2012, 86, 155441. b) Sivan, Y.; Sonnefraud, Y. Plasmonics and Super Resolution Imaging, edited by Zhaowei Liu, to appear ed.; PAN Stanford, 2016.

35 Wolfbeis, O. S. An Overview of Nanoparticles Commonly Used in Fluorescent Bioimaging. Chem. Soc. Revi. 2015, 44, 4743-4768. 
${ }^{36}$ Pustovalov, V. K. Light-to-heat Conversion and Heating of Single Nanoparticles, their Assemblies, and the Surrounding Medium Under Laser Pulses. RSC Adv. 2016, 6, 81266-81289.

${ }^{37}$ Bordenave, M. D.; Francisco, B.; Fernando, D. S.; Stefan, W. H. STED Nanoscopy with Wavelengths at the Emission Maximum. J. Phys. D: Appl. Phys. 2016, 49, 365102.

38 Vicidomini, G.; Moneron, G.; Eggeling, C.; Rittweger, E.; Hell, S. W. STED with Wavelengths Closer to the Emission Maximum. Opt. Express 2012, 20, 5225-5236.

39 Zhou, N.; Yuan, M.; Gao, Y.; Li, D.; Yang, D. Silver Nanoshell Plasmonically Controlled Emission of Semiconductor Quantum Dots in the Strong Coupling Regime. ACS Nano 2016, 10, 4154-4163.

40 Rakovich, A.; Albella, P.; Maier, S. A. Plasmonic Control of Radiative Properties of Semiconductor Quantum Dots Coupled to Plasmonic Ring Cavities. ACS Nano 2015, 9, 2648-2658.

${ }^{41} \mathrm{Han}, \mathrm{K}$. Y.; Ha, T. Dual-Color Three-Dimensional STED Microscopy with a Single High-Repetition-Rate Laser. Opt. Lett. 2015, 40, 2653-2656.

42 Johnson, P. B.; Christy, R. W. Optical Constants of the Noble Metals. Phys. Rev. B 1972, 6, 43704379.

${ }^{43}$ Auksorius, E.; Boruah, B. R.; Dunsby, C.; Lanigan, P. M. P.; Kennedy, G.; Neil, M. A. A.; French, P. M. W. Stimulated Emission Depletion Microscopy with a Supercontinuum Source and Fluorescence Lifetime Imaging. Opt. Lett. 2008, 33, 113-115.

44 Lenz, M. O.; Sinclair, H. G.; Savell, A.; Clegg, J. H.; Brown, A. C. N.; Davis, D. M.; Dunsby, C.; Neil, M. A. A.; French, P. M. W. 3-D Stimulated Emission Depletion Microscopy with Programmable Aberration Correction. J. Biophotonics 2014, 7, 29-36.

45 Vicidomini, G.; Schönle, A.; Ta, H.; Han, K. Y.; Moneron, G.; Eggeling, C.; Hell, S. W. STED Nanoscopy with Time-Gated Detection: Theoretical and Experimental Aspects. PLoS ONE 2013, 8, e54421.

${ }^{46}$ Warren, S. C.; Margineanu, A.; Alibhai, D.; Kelly, D. J.; Talbot, C.; Alexandrov, Y.; Munro, I.; Katan, M.; Dunsby, C.; French, P. M. W. Rapid Global Fitting of Large Fluorescence Lifetime Imaging Microscopy Datasets. PLOS ONE 2013, 8, e70687.

${ }^{47}$ Donehue, J. E.; Wertz, E.; Talicska, C. N.; Biteen, J. S. Plasmon-Enhanced Brightness and Photostability from Single Fluorescent Proteins Coupled to Gold Nanorods. J. Phys. Chem. C 2014, 118, 15027-15035.

48 Pellegrotti, J. V.; Acuna, G. P.; Puchkova, A.; Holzmeister, P.; Gietl, A.; Lalkens, B.; Stefani, F. D.; Tinnefeld, P. Controlled Reduction of Photobleaching in DNA Origami-Gold Nanoparticle Hybrids. Nano Lett. 2014, 14, 2831-2836. 
49 Galloway, C. M.; Artur, C.; Grand, J.; Ru, E. L. Photobleaching of Fluorophores on the Surface of Nanoantennas. J. Phys. Chem. C 2014, 118, 28820-28830.

\section{TOC image:}

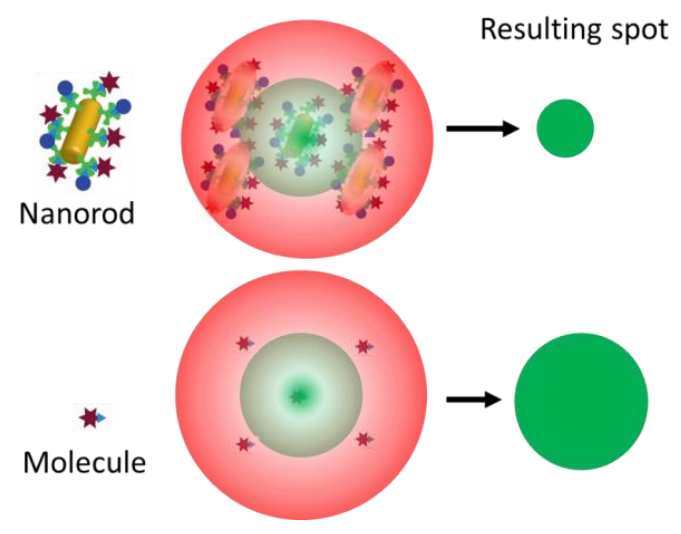

